\title{
Characterisation of LDL receptor gene mutations in a North Indian cohort of children with homozygous familial hypercholesterolaemia
}

Charakterystyka mutacji genów receptora LDL w kohorcie północnoindyjskiej dzieci z homozygotyczną rodzinną hipercholesterolemią

\author{
Shagun Singh, Minu Singh, Devi Dayal, Prateek Bhatia, Sandeep Negi, Savita V. Attri
}

Department of Pediatrics, Postgraduate Institute of Medical Education and Research, India

\begin{abstract}
Background: Homozygous familial hypercholesterolaemia $(\mathrm{HoFH})$ carries a grave prognosis but is often underdiagnosed and undertreated. Confirmation of molecular diagnosis helps in planning effective management and determining prognosis accurately.

Aim of the study: To determine the spectrum of mutations in the LDLR gene in a cohort of children with a clinical diagnosis of HoFH. Material and methods: Genomic DNA was extracted from peripheral blood samples of 8 patients, who were children of either sex, aged under 16 years, and diagnosed clinically with HoFH using the Simon Broome criteria. The potential variants in the $L D L R$ gene were analysed by Sanger sequencing.

Results: Fifty variations were found in the 8 patients; 39 (78\%) were single nucleotide variations while 8 (16\%) and 3 (6\%) were deletions and insertions, respectively. The pathogenic variants in the LDLR gene were detected in four patients; three showed duplication in exon 17 (c.2416dupG) creating an amino acid change at position 806 (p.Val806GlyfsTer11) while one had a missense variant in the exon 9 at position c.1285G>A resulting in a change in amino acid at position 429 (p.Val429Met). The variants were found in heterozygous state in the parents or siblings of probands who showed pathogenic variants.

Conclusions: The frequency of disease-causing variants in the LDLR gene in our patients with HoFH was $50 \%$. Further studies to characterise mutations in genes for apolipoprotein B, proprotein convertase subtilisin/kexin type 9, or LDL adaptor protein are suggested in all children with a clinical diagnosis of $\mathrm{HoFH}$.
\end{abstract}

Key words:

homozygous familial hypercholesterolaemia, LDLR gene, pathogenic variants, Indian families.

\section{Introduction}

Familial hypercholesterolaemia $(\mathrm{FH})$ is an inherited disorder of plasma lipoprotein metabolism characterised by markedly increased and sustained plasma low-density lipoprotein cholesterol (LDL-C) concentrations, which increase the risk of premature atherosclerotic cardiovascular disease (CVD) in the affected individuals by 3 - to 10 -fold versus the general population [1]. Of the two $\mathrm{FH}$ types, heterozygous $\mathrm{FH}(\mathrm{HeFH})$ is milder but more frequent, with a prevalence of $1 / 500$ to $1 / 200$, whereas the homozygous form $(\mathrm{HoFH})$ is severe but less prevalent (about $1 / 1,000,000$ ) [1]. The HoFH is caused by mutations in the genes for either LDL Receptor (LDLR) protein, apolipoprotein B (APOB), proprotein convertase subtilisin/kexin type 9 (PCSK9), or LDL adaptor protein (LDLRAP) [2]. About 85\% of patients with $\mathrm{HoFH}$ have mutations in the $L D L R$ gene, located on chromosome 19. The LDLR is a transmembrane protein on the surface of hepatocytes, which mediates the uptake of plasma LDL into hepatocytes for further metabolism. Mutations in the $L D L R$ gene either reduce the number or function of LDLR molecules, which results in decreased clearance of LDL. As a consequence, the plasma concentrations LDL as well as total cholesterol (TC) increase. The plasma concentrations of lipoprotein particles, predominantly LDL, are critical determinants for initiating changes in the vascular endothelium, which result in atherosclerosis and premature $\mathrm{CAD}$ as well as valvular lesions and peripheral arterial disease [1, 2].

Despite a grave prognosis, $\mathrm{FH}$ remains underdiagnosed and undertreated; only about $20 \%$ of patients are diagnosed during childhood. The clinical diagnosis is usually based on one of the several proposed criteria, such as the Simon Broome Register Group criteria, Dutch Lipid Clinic Network criteria, Make Early Diagnosis to Prevent Early Deaths (MEDPED) criteria, the Japanese criteria, and the Simplified Chinese 
Criteria for Familial Hypercholesterolaemia (SCCFH) [2, 3]. However, the clinical diagnostic criteria for $\mathrm{FH}$ can be inaccurate and often need to be supported by genetic diagnosis for several reasons. The characterisation of genetic defect allows cascade testing, has prognostic utility and allows determination of response to lipid lowering drugs [2]. For example, the homozygous patients show higher basal LDL-C concentrations and a poorer response to therapy compared with compound heterozygotes [2]. The response to statins is limited in patients with homozygous or compound heterozygous for damaging or null $L D L R$ variants because these drugs remove circulating LDL and remnant lipoproteins by increasing the LDLR expression on the cell surface [4]. The statins may exacerbate the disease in cases of $\mathrm{FH}$ caused by lysosomal acid lipase deficiency by increasing the endocytosis of cholesteryl esters. Drugs that do not require a competent LDLR for their action such as bile acid ion-exchange resins, niacin, ezetimibe, and lomitapide are effective in FH due to mutations in ATP binding cassette subfamily G [4]. Lastly, molecular diagnosis of $\mathrm{HoFH}$ may help to avoid situations where xanthoma is misdiagnosed as other skin lesions and therefore mistreated in clinical practice $[5,6]$

In many developed countries, genetic screening is routinely performed in families with $\mathrm{FH}$, especially $\mathrm{HoFH}$, which has helped in the characterisation of prevalent mutations in their populations and allowed the development of cost-effective diagnostic evaluation $[7,8]$. In India, however, children with $\mathrm{FH}$ are usually diagnosed and treated based on clinical criteria, and there are only limited data on the genetic diagnosis of $\mathrm{FH}[9]$. One of the three previous genetic studies in children with $\mathrm{HoFH}$ was conducted on a patient population from Southwest India [10]. The other two studies from Northwest India observed that the spectrum of mutations in the Asian Indian population was quite heterogeneous [11, 12]. With India being a multi-ethnic country, wide variations are expected in the genetic mutations affecting patient populations in different regions of the country [13]. We therefore planned to determine the spectrum of mutations in the LDLR gene in our cohort of North Indian children with a clinical diagnosis of $\mathrm{HoFH}$, and their parents.

\section{Material and Methods}

\section{Study population}

The probands $(n=8)$ were children of either sex, aged less than 16 years, and diagnosed clinically with $\mathrm{HoFH}$ using the Simon Broome criteria - briefly, presence of cutaneous or tendinous xanthomas, and/or plasma TC and LDL-C concentrations $>260 \mathrm{mg} / \mathrm{dl}(6.7 \mathrm{mmol} / \mathrm{l})$ and $>155 \mathrm{mg} / \mathrm{dl}(4.0 \mathrm{mmo} / \mathrm{l})$, respectively. Two family members of the probands, mainly parents also participated in the study. The study participants gave written informed consent prior to their enrolment, which adhered to the World Medical Association Declaration of Helsinki. Children were included with parental consent and their own assent wherever applicable. The study was approved by the Institute's Ethics Committee (INT/IEC/2020/000064).
Biochemical analyses, and extraction, amplification and sequencing of genomic DNA

After overnight fasting, blood from the study participants was obtained in tubes containing $0.1 \%$ ethylenediaminetetraacetic acid, and plasma was separated. Measurement of TC high-density lipoprotein cholesterol (HDL-C), and triglycerides (TG) was done on an automated chemical analyser (Advia 1800 clinical chemistry analyser, Siemens Healthcare Diagnostics, USA). The LDL-C fraction was calculated by Friedewald equation as: LDL-C $(\mathrm{mg} / \mathrm{dl})=\mathrm{TC}-\mathrm{HDL}-\mathrm{C}-(\mathrm{TG} / 5)$. Genomic DNA was extracted from blood using a QIAmp DNA blood kit (Qiagen). Specific primers for the 18 exons of the $L D L R$ gene (RefSeq Accession: NG 00060) were designed using the Primer3www.bioinformatics.nl server. The primers designed in the adjoining intron regions flanked the whole exon. The extracted DNA samples were quantified using spectrophotometer (NanoDrop). The quality of DNA was checked based on the 260/280 absorption ratio, and the samples with ratios around 1.8 were selected for further amplification using PCR. The PCR amplicons were then purified using ExoSAP-IT (ThermoFisher Scientific) in a thermal cycler according to the manufacturer's protocol. The amplicons and the primers were then submitted for Sanger Sequencing (Abi 3500 Genetic Analyzer) and the results were analysed using Automated Mutation Analysis Pipeline, and the reported variations were checked in the $L D L R$ gene database (http://www.lovd.nl/LDLR). The pathogenicity of variants was analysed using bioinformatics software tools such as PolyPhen-2, SIFT, and Mutation Taster and Variant Effect Predictor (VEP) using plug-ins dbscSNV and MaxEntScan for splice site variants. Mean allele frequency was checked on the gnomAD database version 2.1.1. The variants were classified according to the American College of Medical Genetics guidelines based on a 5-tier system [14].

\section{Statistical analysis}

Data were analysed using Statistical Package for the Social Sciences (SPSS, version 23.0, IBM Corp., Armonk, NY, USA). Descriptive statistics were used for baseline comparison. The genotype phenotype relationship was determined by using univariate factors such as age, presence of xanthomas, LDL levels, and the gene variants. Spearman's rank correlation coefficient was determined with each factor. The existence of Hardy-Weinberg equilibrium was analysed using gene frequencies obtained by simple gene counting, and the chi-square test with Yates' correction was applied for comparing observed and expected values. A $p$-value of $<0.05$ was considered statistically significant.

\section{Results}

Clinical characteristics of the study cohort

Eight families belonging to three major states of Northwest India, i.e. Punjab (5 families), Haryana (2 families), and Himachal Pradesh ( 1 family), were included in this study. Six probands were boys and 2 were girls; their mean age at diagnosis was 6.2 \pm 2.6 years (range 3-9 years). All patients except 2 presented 
with xanthomas. Family history of early CVD was not present in any patient; one parent was on treatment for elevated TC. The mean concentrations of TC, TG, and LDL-C were $548.7 \pm 180.7$, $318.2 \pm 471.2$, and $417.0 \pm 162.6 \mathrm{mg} / \mathrm{dl}$, respectively. The mean carotid intima media thickness was $0.37 \pm 0.06 \mathrm{~mm}$ (normal reference values $0.34 \pm 0.05 \mathrm{~mm}$, cut-off $>0.45 \mathrm{~mm}$ ) [15].

\section{Genetic analysis}

A total of 50 variations were found in the 8 patients; 23 (46\%) were non-coding, 19 (38\%) synonymous, and 4 (8\%) frameshift and missense variants. A majority of the variants (39, $78 \%)$ were single nucleotide variations (SNV), while $8(16 \%)$ and $3(6 \%)$ were deletions and insertions, respectively.

Four patients $(50 \%)$ showed $\mathrm{HoFH}$-causing variants in the LDLR gene (Table I). The other four did not show any clinically relevant mutation. Three out of four patients contained pathogenic variant (duplication) in exon 17 (c.2416dupG) creating an amino acid change at position 806 (p.Val806GlyfsTer11), leading to protein chain termination following 11 amino acids. The other variant (missense) was noted in exon 9 of the $L D L R$ gene at position $\mathrm{c} .1285 \mathrm{G}>\mathrm{A}$. This mutation leads to the change in amino acid at position 429 (p.Val429Met) (Fig. 1). All the abovenoted variants were tested in the parent's or sibling's DNA sample and were detected in heterozygous state in each case.

\section{Genotype-phenotype relationship}

We did not find any relationship between the genotype and phenotype in our patients. The presence of pathogenic variants did not show any association to the age $\left(\chi^{2}=0.0, p\right.$-value 1.0), presence of xanthoma $\left(\chi^{2}=0.533, p\right.$-value 0.465$)$, and concentrations of LDL-C $\left(\chi^{2}=4.8, p\right.$-value 0.91$)$ and TC $(0.533$, p-value 0.465).

\section{Discussion}

In our observational study, we characterised disease-causing mutations in the $L D L R$ gene in 8 children with a definite or possible clinical diagnosis of $\mathrm{HoFH}$. The pathogenic variants were found in 4 (50\%) patients, 3 of whom had the same variant. In other cohorts of $\mathrm{HoFH}, L D L R$ gene mutations have been reported with a frequency ranging between 80 and al- most $100 \%[1,2,11]$. It is known that the range of $L D L R$ variants changes between populations because approximately 1700 different mutations have been identified around the world (www.ucl.ac.uk/ldlr). Similarly, the proportion of true versus compound heterozygotes was $50 \%$ as compared to other cohorts that have very few true homozygotes [2].

The insertion observed in 3 patients at position 211 in exon 17 that resulted in duplication of $G$ (c.2416dupG (p.Val806GlyfsTer11) causes the reading frame to shift at codon 806 , changing valine to glycine and creating a premature stop codon [16]. This results in a truncated, abnormal protein product or loss of protein through nonsense-mediated mRNA decay. In one family, this variant was also found in a heterozygous state in the father, who had elevated LDL levels; the data for the mother could not be interpreted due to poor readings. In the second patient, the mother and a sibling (brother) were found to be heterozygous for the same mutation; father's sample was not available for analysis. The sequencing data for the parents of the third child could be confirmed only in the mother, who had the same heterozygous variant. This mutation has been identified in patients from different ethnic backgrounds such as Japanese, Pakistani, and Indian [11, 17, 18].

Only one of our patients showed a mutation in exon 9, which is considered as one of the hot spots in studies performed on Asian Indians in South Africa [11]. This missense variant at position 11224052 results in replacement of valine with methionine at codon 429 of the LDLR protein (p.Val429Met) and causes a slower processing of the precursor LDLR protein to its mature form and hence its faster degradation [19]. The mother of the proband had the same heterozygous variant; however, it could not be identified in the father's DNA. This variant has been reported in patients belonging to different ethnicities. It was found in 4 of 28 Greek patients, 2 of 1070 Italian individuals, and 161 members of a large Dutch family with $\mathrm{FH}$ [20-22].

We did not find any new variants in the $L D L R$ gene in our cohort of HoFH children. However, the detected variants in our patients are different from previous studies from Northwest India, suggesting a different lineage or ancestry of our cohort despite belonging to a similar geographic location. This is explained by the genetic heterogeneity due to the multi-ethnic

Table I. Pathogenic mutations in the LDLR gene detected in 4 patients and their corresponding lipid levels

\begin{tabular}{llllllll}
\hline Patient & Genetic status & Exon & Nucleotide change & Protein change & RefSNP & TC (mg/dl) & LDL-C (mg/dl) \\
\hline A & Homozygous & 17 & c.2416dupG & p.Val806GlyfsTer11 & rs773618064 & 550 & 415 \\
\hline N & Homozygous & 17 & c.2416dupG & p.Val806GlyfsTer11 & rs773618064 & 552 & 376 \\
\hline B & Homozygous & 9 & c.1285G $>$ A & p.Val429Met & rs28942078 & 530 & 485 \\
\hline D & Homozygous & 17 & c.2416dupG & p.Val806GlyfsTer11 & rs773618064 & 651 & 534 \\
\hline
\end{tabular}

RefSNP - reference single nucleotide polymorphism; TC - total cholesterol; LDL-C - low-density lipoprotein cholesterol 
composition of the Indian population [13]. The limitations of our study were the small sample size, which was primarily due to financial constraints, and the inability to perform a robust mutation segregation analysis for all patients due to missing data of either parents or siblings.
In conclusion, the disease-causing variants in the $L D L R$ gene were detected in only $50 \%$ of our patients with $\mathrm{HoFH}$. Larger studies are recommended to characterise the variants in the genes other than the LDLR gene in patient populations with $\mathrm{HoFH}$.

Exon 17: c.2416dupG (Val806Gly)

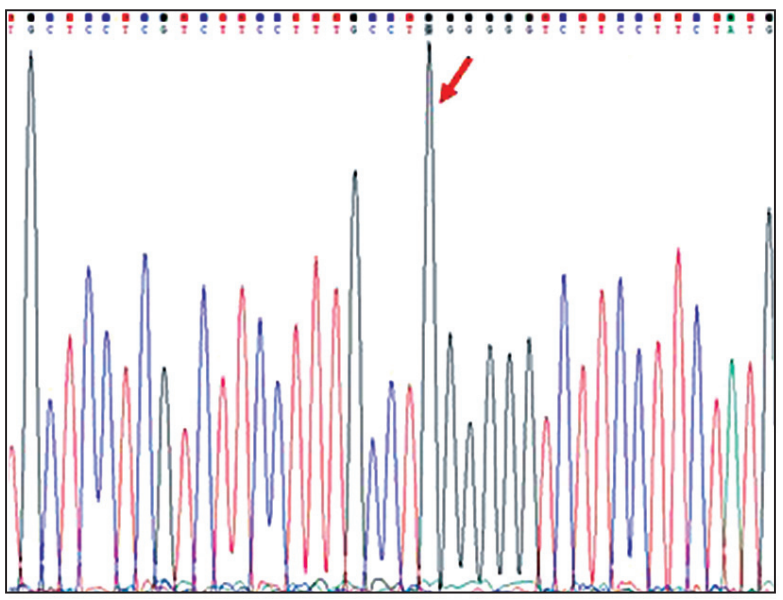

Proband

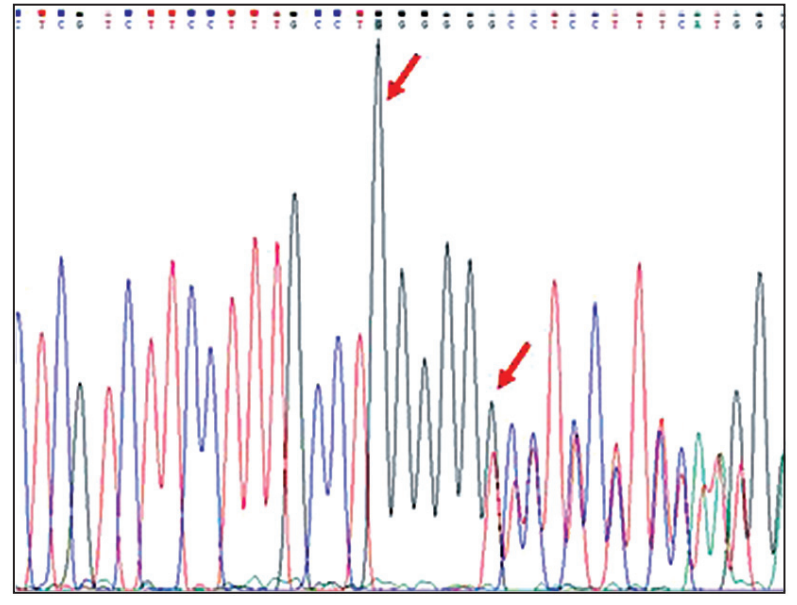

Father

Exon 9: c.1285G >A (Val429Met)

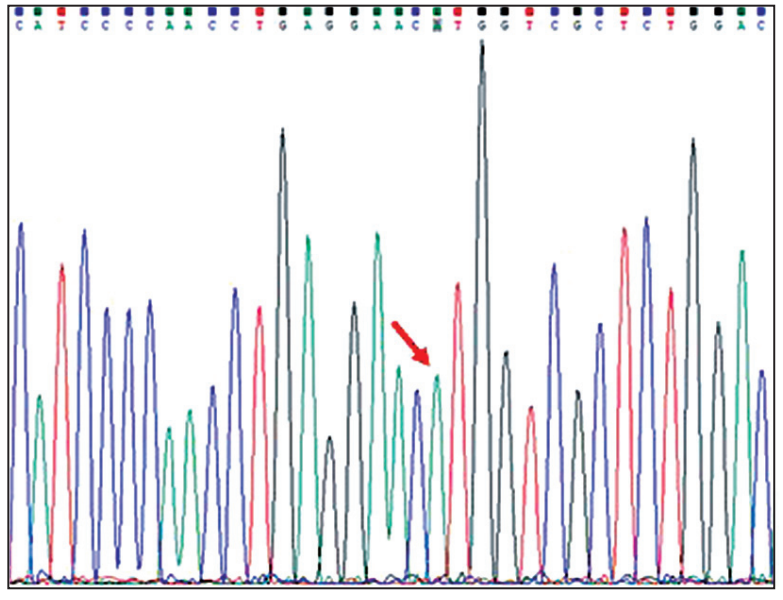

Proband

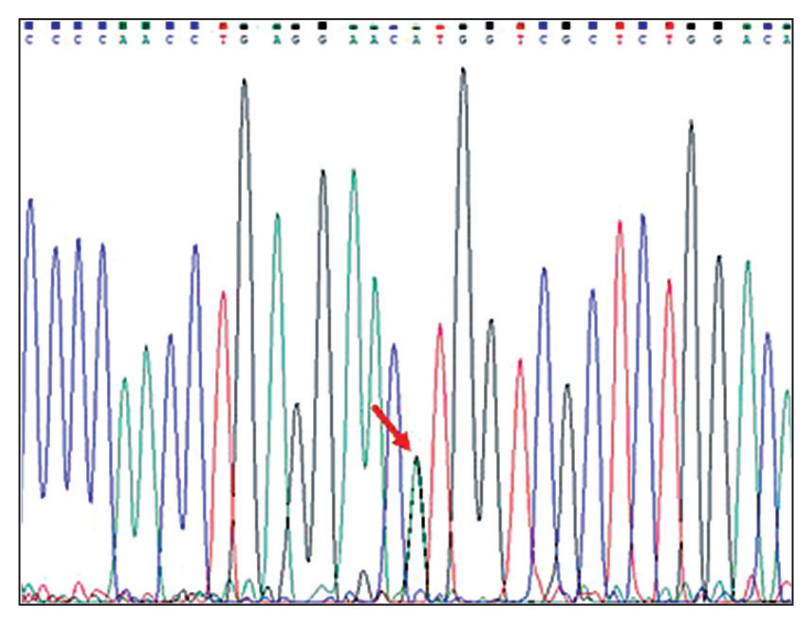

Mother

Figure 1. Representative chromatograms of exon 17: c.2416dupG (Val806Gly) and exon 9: c.1285G>A (Val429Met) variants in two probands and their respective parents 


\section{References}

1. Singh S, Bittner V. Familial hypercholesterolemia - epidemiology, diagnosis, and screening. Curr Atheroscler Rep 2015; 17: 482. doi: 10.1007/s11883-014-0482-5

2. Di Taranto MD, Giacobbe C, Buonaiuto A, et al. A Real-World Experience of Clinical, Biochemical and Genetic Assessment of Patients with Homozygous Familial Hypercholesterolemia. J Clin Med 2020; 9: E219. doi: 10.3390/jcm9010219

3. Cao YX, Sun D, Liu HH, et al. A Novel Modified System of Simplified Chinese Criteria for Familial Hypercholesterolemia (SCCFH). Mol Diagn Ther 2019; 23: 547-553. doi: 10.1007/s40291-019-00405-1

4. Raal FJ, Hovingh GK, Catapano AL. Familial hypercholesterolemia treatments: Guidelines and new therapies. Atherosclerosis 2018; 277: 483-492. doi: 10.1016/j.atherosclerosis.2018.06.859

5. Randhawa MS, Varma TH, Dayal D. Benign calcinosis cutis. Turk Pediatri Ars 2018; 53: 267-268. doi: 10.5152/TurkPediatriArs.2018.6792

6. Alnouri F, Al-Allaf FA, Athar M, et al. Xanthomas Can Be Misdiagnosed and Mistreated in Homozygous Familial Hypercholesterolemia Patients: A Call for Increased Awareness Among Dermatologists and Health Care Practitioners. Glob Heart 2020; 15: 19. doi: 10.5334/gh.759

7. Humphries SE, Cooper J, Dale P, et al.; FH Paediatric Register Steering Group. The UK Paediatric Familial Hypercholesterolaemia Register: Statin-related safety and 1-year growth data. J Clin Lipidol 2018; 12: 25-32. doi: 10.1016/j.jacl.2017.11.005

8. Hernández Flores TJ, González García JR, Sánchez López YJ, et al. LDLR Gene Mutation p.Asp360His and Familial Hypercholesterolemia in a Mexican Community. Arch Med Res 2020; 51 : 153-159. doi: 10.1016/j.arcmed.2019.12.017

9. Dayal D, Seetharaman K, Bhunwal S, et al. Long-term use of a combination of atorvastatin and ezetimibe in children with homozygous familial hypercholesterolemia. Int J Contemp Pediatr 2018; 5: 275-277. doi: 10.1017/S1047951115000591

10. Kulkarni SS, Basavraj S, Kadakol GS, et al. Mutation Analysis of the LDL Receptor Gene in Indian Families with Familial Hypercholesterolemia. Asian J Med Sci 2011; 2: 82-86.

11. Setia N, Saxena R, Arora A, et al. Spectrum of mutations in homozygous familial hypercholesterolemia in India, with four novel mutations. Atherosclerosis 2016; 255: 31-36. doi: 10.1016/j.atherosclerosis.2016.10.028

12. Setia N, Saxena R, Sawhney JPS, et al. Familial Hypercholesterolemia: Cascade Screening in Children and Relatives of the Af- fected. Indian J Pediatr 2018; 85: 339-343. doi: 10.1007/s12098017-2589-5

13. Basu A, Sarkar-Roy N, Majumder PP. Genomic reconstruction of the history of extant populations of India reveals five distinct ancestral components and a complex structure. Proc Natl Acad Sci U S A 2016; 113: 1594-1599. doi: 10.1073/pnas.1513197113

14. Richards S, Aziz N, Bale S, et al.; ACMG Laboratory Quality Assurance Committee. Standards and guidelines for the interpretation of sequence variants: a joint consensus recommendation of the American College of Medical Genetics and Genomics and the Association for Molecular Pathology. Genet Med 2015; 17: 405-424. doi: 10.1038/gim.2015.30

15. Sajja V, Jeevarathnam D, James S, Rathinasamy J. A study on carotid artery intima-media thickness and metabolic risk factors in overweight and obese Indian children. Diabetol Int 2019; 11: 142149. doi: 10.1007/s13340-019-00417-6

16. Nicholls P, Young I, Lyttle K, Graham C. Screening for familial hypercholesterolaemia. Early identification and treatment of patients is important. BMJ 2001; 322: 1062.

17. Yu W, Nohara A, Higashikata T, et al. Molecular genetic analysis of familial hypercholesterolemia: spectrum and regional difference of LDL receptor gene mutations in Japanese population. Atherosclerosis 2002; 165: 335-342. doi: 10.1016/s0021-9150(02)00249-6

18. Ajmal M, Ahmed W, Akhtar N, et al. A Novel Pathogenic Nonsense Triple-Nucleotide Mutation in the Low-Density Lipoprotein Receptor Gene and Its Clinical Correlation with Familial Hypercholesterolemia. Genet Test Mol Biomark 2011; 15: 601-606. doi: 10.1089/ gtmb.2010.0184

19. Leitersdorf E, Van der Westhuyzen DR, Coetzee GA, et al. Two common low density lipoprotein receptor gene mutations cause familial hypercholesterolemia in Afrikaners. J Clin Invest 1989; 84: 954-961. doi: 10.1172/JCl114258

20. Usifo E, Leigh SEA, Whittall RA, et al. Low-Density Lipoprotein Receptor Gene Familial Hypercholesterolemia Variant Database: Update and Pathological Assessment. Ann Hum Genet 2012; 76 : 387-401. doi: 10.1111/j.1469-1809.2012.00724.x

21. Bertolini S, Pisciotta L, Rabacchi C, et al. Spectrum of mutations and phenotypic expression in patients with autosomal dominant hypercholesterolemia identified in Italy. Atherosclerosis 2013; 227 : 342-348. doi: 10.1016/j.atherosclerosis.2013.01.007

22. Versmissen J, Oosterveer DM, Yazdanpanah $M$, et al. Identifying genetic risk variants for coronary heart disease in familial hypercholesterolemia: an extreme genetics approach. Eur J Hum Genet 2015; 23: 381-387. doi: 10.1038/ejhg.2014.101 